\title{
Retinal projection type super multi-view 3D head-mounted display using the time division projection optical system
}

\section{Tadayuki Konda, Katsuhisa Tanaka, Kayo Yoshimoto, Hideya Takahashi}

\begin{tabular}{|c|l|}
\hline Citation & Proceedings Volume 10556, Advances in Display Technologies VIII; 105560L \\
\hline Issue Date & 2018-02-19 \\
\hline Type & Conference Paper \\
\hline Textversion & Author \\
\hline Description & Event: SPIE OPTO, 2018, San Francisco, California, United States \\
\hline Rights & $\begin{array}{l}\text { C (2018) COPYRIGHT Society of Photo-Optical Instrumentation Engineers } \\
\text { (SPIE). One print or electronic copy may be made for personal use only. } \\
\text { Systematic reproduction and distribution, duplication of any material in this } \\
\text { publication for a fee or for commercial purposes, and modification of the } \\
\text { contents of the publication are prohibited. }\end{array}$ \\
\hline Relation & $\begin{array}{l}\text { The following paper is an author-prepared version or an official version } \\
\text { (preferred version) of the publication. After it is published, it will be found at } \\
\text { https://doi.org/10.1117/12.2287649. }\end{array}$ \\
\hline DOI & \begin{tabular}{l}
$10.1117 / 12.2287649$ \\
\hline
\end{tabular} \\
\hline
\end{tabular}

\author{
Self-Archiving by Author(s) \\ Placed on: Osaka City University
}

Tadayuki Konda, Katsuhisa Tanaka, Kayo Yoshimoto, and Hideya Takahashi "Retinal projection type super multi-view 3D head-mounted display using the time division projection optical system", Proc. SPIE 10556, Advances in Display Technologies VIII, 105560L (19 February 2018); 


\title{
Retinal projection type super multi-view 3D head-mounted display using the time division projection optical system
}

\author{
Tadayuki Konda ${ }^{\mathrm{a}}$, Katsuhisa Tanaka ${ }^{\mathrm{a}}$, Kayo Yoshimoto ${ }^{\mathrm{a}}$, and Hideya Takahashi ${ }^{* a}$ \\ ${ }^{a}$ Dept. of Electrical and Information Engineering, Graduate School of Engineering, Osaka City Univ., \\ 3-3-138 Sugimoto, Sumiyoshi-ku, Osaka 558-8585, Japan;
}

\begin{abstract}
We have previously proposed the see-through retinal projection type super multi-view head-mounted display (HMD). The smooth motion parallax provided by the super multi-view technique enables a precise superposition of virtual 3D images on real scene. Moreover, if a viewer focuses one's eyes on the displayed 3D image, the stimulus for the accommodation of human eye is reproduced naturally. To realize the super multi-view condition, multiple parallax images must be projected onto the retina. However, in the previous proposed HMD, since the respective parallax images were spatially divided and were projected onto the retina, the image resolution was low and the optical system was complicated. In order to overcome these problems, we propose the improved see-through retinal projection type super multi-view HMD by using the time division projection optical system. The proposed HMD consists of a multiple exposure holographic lens with multi-convergence points, a high frame rate display device, and a high-speed optical shutter. Multiple parallax images are displayed by time division and are converged on respective points by the holographic lens. The optical shutter which synchronized to the display device passes only one convergence light corresponding to the right parallax image. Therefore, proposed HMD realizes the pseudo super multi-view condition and displays the virtual image at the distance within ability for focusing on the human eye. To verify the effectiveness of the proposed HMD, we confirmed the depth range of the 3D image by the prototype of the proposed HMD was more than $250 \mathrm{~mm}$ in front of the pupil.
\end{abstract}

Keywords: retinal projection display, super multi-view, head-mounted display, 3D display

\section{INTRODUCTION}

Mixed Reality technology ${ }^{1}$ has recently been proposed as an approach for practical use of Virtual Reality technology ${ }^{2}$ See-through head-mounted displays (HMD) provide an effective capability for Mixed Reality. By using a see-through HMD, an observer can see both real world and virtual world at the same time. Some conventional see-through HMDs have been developed. They can display two-dimensional virtual information. However, when two-dimensional virtual information is displayed by a HMD, an observer is hard to understand that it is related with a real-world object. What an observer sees needs to be augmented by three-dimensional (3D) virtual information image in accordance with the real object. In order to overcome this problem, we have previously proposed a retinal projection type super multi-view $\mathrm{HMD}^{3-5}$. The smooth motion parallax provided by the super multi-view technique ${ }^{6}$ enables a precise superposition of virtual 3D images on real objects. Moreover, if a viewer focuses his or her eyes on the displayed 3D image, the stimulus for the accommodation of the human eye is reproduced naturally by the super multi-view technique. Therefore, although the previously proposed HMD is a monocular HMD, it provides observers with natural 3D images. In addition, the previously proposed retinal projection type super multi-view HMD use the principle of the Maxwellian view ${ }^{7}$. In the Maxwellian view, parallel rays are converged directly at the center of the pupil, and projected onto the retina directly. Thus, previously proposed HMD can provide an extreme long focal depth image, and a provided 3D image by the proposed HMD is clear and high contrast. However, in the previous proposed HMD, since the respective parallax images were spatially divided and were projected onto the retina, the image resolution was low and the optical system was complicated $^{3,4}$. In order to overcome these problems, we propose the improved see-through retinal projection type super multi-view HMD by using the time division projection optical system. This paper describes the principle of the improved retinal projection type super multi-view HMD. In Section 2, we describe the principle of the Maxwellian view, the principle of the previously proposed retinal projection type super multi-view HMD and its drawback. In Section 3 , the

*hideya@elec.eng.osaka-cu.ac.jp; 
principle of the proposed improved see-through retinal projection type super multi-view HMD by using the time division projection optical system is described. In Section 4, to verify the effectiveness of the improved HMD, we describe experimental result. Finally, we give some conclusions in Section 5.

\section{PREVIOUSLY PROPOSED RETINAL PROJECTION TYPE SUPER MULTI-VIEW HMD}

We have previously proposed a see-through retinal projection type 3D $\mathrm{HMD}^{7-9}$. In this paper, since we describe improvement of the previously proposed HMD, the previously proposed see-through retinal projection type 3D HMD is described here again.

\subsection{Holographic Combiner}

The fundamental role of an HMD is to produce a distinguishable image of the Spatial Light Modulator (SLM) and enable the outside world to be seen through the see-through HMD. In our previous HMD, we used an HOE as a combiner which superimposes the virtual image on the real scene ${ }^{8-10}$. Figure 1 shows the principle of a holographic combiner. The HOE is a diffraction grating which is made by using the holography technique. In our previous HMD, the HOE has two functions. One is a half mirror to combine the real world and a virtual image, and the other is a lens to achieve the Maxwellian view. And this HOE has sufficient transparency to see the outside world because it has superior optical characteristics such as wavelength selectivity and angular selectivity. Moreover, since the HOE consists of a thin layer of emulsion, it contributes to compact mobility and lightweight of an HMD.

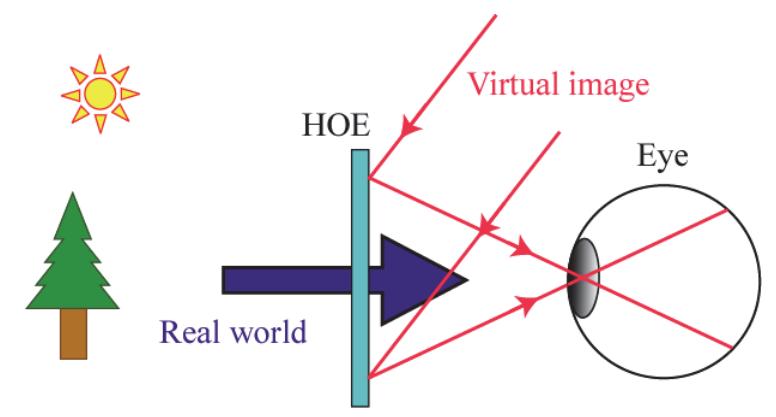

Figure 1. Principle of a holographic combiner.

\subsection{Principle of the Maxwellian View}

The Maxwellian view is the key technology of our previous HMD ${ }^{5}$. In the Maxwellian view, parallel rays are converged directly at the center of the pupil, and projected onto the retina directly. This technique is used to measure the sensitivity of the human vision system or in experiments of psychological visual perception. Figure 2 shows the principle of the Maxwellian view. The parallel rays irradiate the transparent object $\mathrm{M}$. The object $\mathrm{M}$ is located on the front focus plane of the lens, and the pupil of the human eye is located on the back focus plane of the lens. In this case, object M and the retina are conjugate, and the light stimulus, which has an extremely long focal depth, can be observed. By using this principle for a retinal projection display, it can provide an extremely long focal depth image without causing ocular accommodation. 


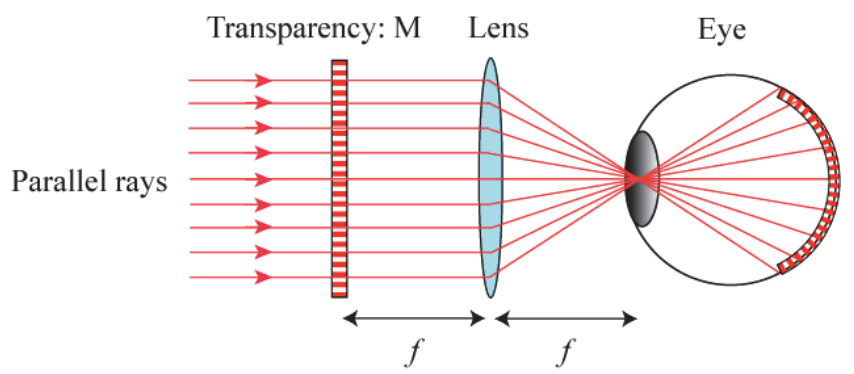

Figure 2. Principle of the Maxwellian view.

\subsection{Super multi-view}

In the real world, usually illumination light is diffusely reflected at an object's surface and reflected light causes stimulus of accommodation of the eye, as shown in Figure 3(a). In the ray reconstruction method, several pixels that have been angularly multiplexed can be utilized to give the impression that rays are emanating from a converging point, as shown in Figure 3(b). However, multiple rays of the light diffusing at the object's surface are sampled discretely. If the sampling interval of parallax rays is narrower than the pupil's diameter, two or more rays always pass through the pupil of each eye. This condition is called 'super multi-view'. In the super multi-view condition, reconstructed 3D images have very smooth motion parallax, and if a viewer focuses his or her eyes on the reconstructed object's surface, the stimulus for the accommodation of the human eye is reproduced naturally. Therefore, the retinal projection HMD provides observers with natural 3D virtual images so long as the super multi-view condition is satisfied.

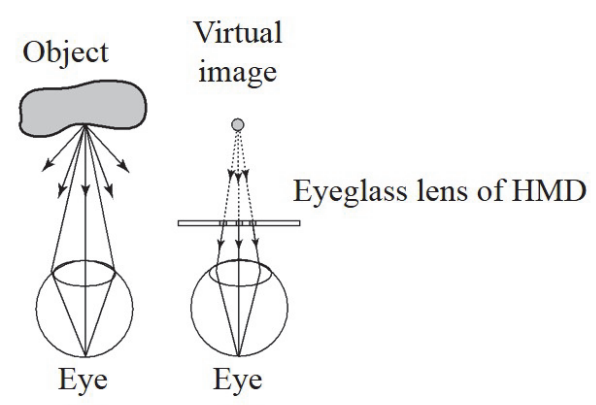

(a) (b)

Figure 3. Schematic diagram of the stimulus of accommodation: (a) real world, (b) super multi-view.

\subsection{Principle of super multi-view HMD}

The proposed retinal projection type super multi-view HMD uses the principle of the Maxwellian view. In the Maxwellian view, parallel rays are converged directly at the center of the pupil. In the proposed HMD, the super multiview condition is realized by some projected parallax images which converge on the different positions on the pupil, as shown in Figure 4. For the sake of simplicity, here we limit the number of parallax images to three. The parallax image $\# i$ converges on the convergent point $C_{i} . P_{i}$ and $Q_{i}$ denote pixels of the parallax image \#i which correspond to virtual $3 \mathrm{D}$ points $\mathrm{P}$ and Q, respectively. In Figure 5(a), when the viewer adjusts the focal length of eyes to match with the spatial position $\mathrm{P}$ naturally then projected image of pixels $\mathrm{P}_{1}, \mathrm{P}_{2}$ and $\mathrm{P}_{3}$ are focused to same position on the retina. The viewer feels this image as the 3D point. On the other hand, when the viewer adjusts the focal length of eyes to match with the spatial position $\mathrm{P}$ then projected image of pixels $\mathrm{Q}_{1}, \mathrm{Q}_{2}$ and $\mathrm{Q}_{3}$ are focused to different positions on the retina. The viewer feels this image is blurred. Similarly, when the viewer adjusts the focal length of eyes to match with the spatial position $\mathrm{Q}$ naturally then projected image of pixels $\mathrm{Q}_{1}, \mathrm{Q}_{2}$ and $\mathrm{Q}_{3}$ are focused to same position on the retina, and projected image of pixels $\mathrm{P}_{1}, \mathrm{P}_{2}$ and $\mathrm{P}_{3}$ are focused to different positions on the retina as shown in Figure 5(b). Thus, the viewer feels that the image of $\mathrm{Q}$ is in focus and the image of $\mathrm{P}$ is out of focus. Therefore, the monocular $3 \mathrm{D}$ display is realized by the monocular parallax in an eye. Since the stimulus of accommodation of the human eye is naturally by the 
super multi-view technique, the proposed HMD displays the virtual image at the distance within the ability for focusing on the eye.

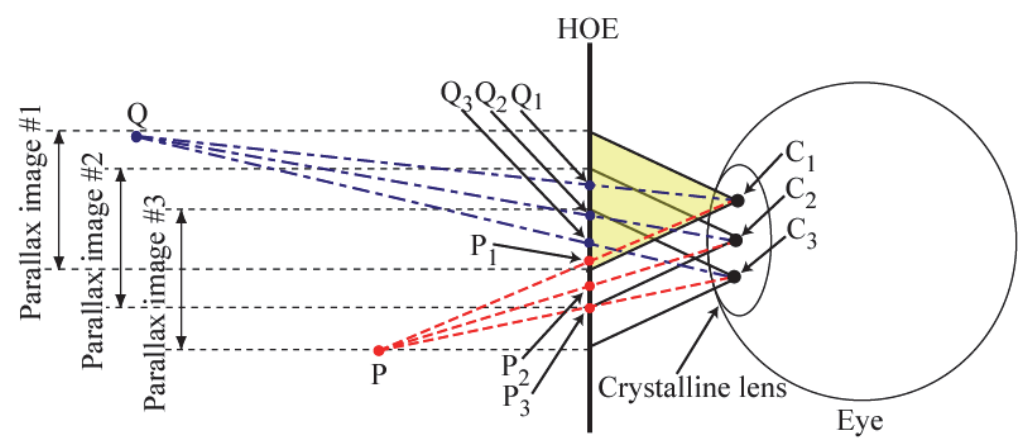

Figure 4. Schematic diagram of the proposed HMD. The super multi-view condition is realized by some projected parallax images which converge on the different positions on the pupil.

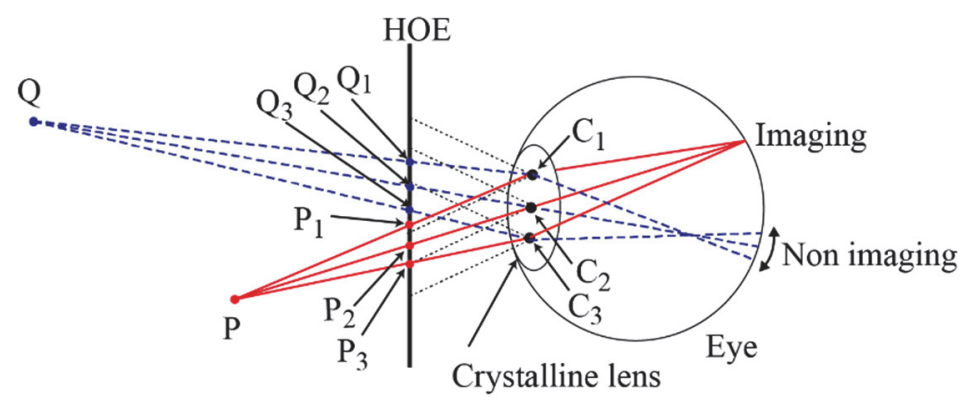

(a)

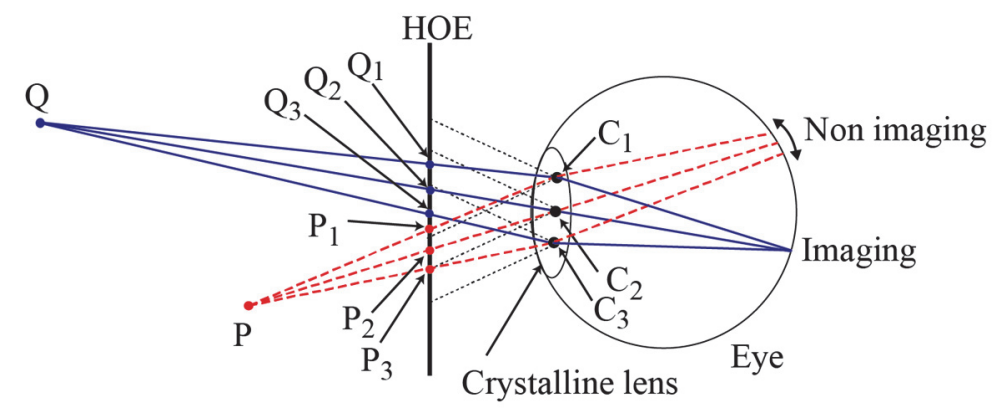

(b)

Figure 5. Principle of the monocular 3D HMD by using the super multi-view technique: (a) focusing on nearby point $\mathrm{P}$, (b) focusing on far-off point $\mathrm{Q}$. 


\subsection{Previous HOE}

In the previous HMD, the number of parallax images is three, thus the number of convergent points on the pupil is also three. Figure 6 shows the structure of the previous HOE. The whole HOE consists of three types of sub-HOEs, $\mathrm{H}_{1}, \mathrm{H}_{2}$ and $\mathrm{H}_{3}$. Each sub-HOE, $\mathrm{H}_{\mathrm{i}}$, converges the corresponding projected parallax image on the convergent point $\mathrm{C}_{\mathrm{i}}$. The whole HOE is composed of stripe structured three types of HOEs as shown in Figure 6. However, since the previous HOE has complicated structured, it is difficult to improve the HOE to increase the number of rays which realize the super multiview condition.

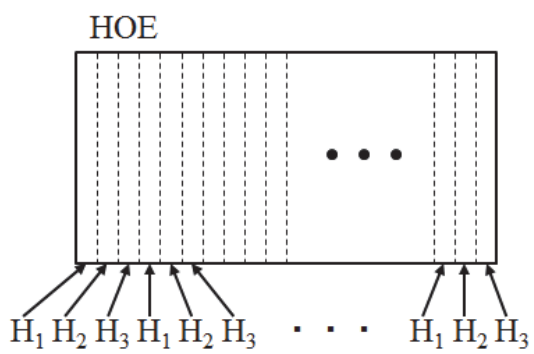

(a)

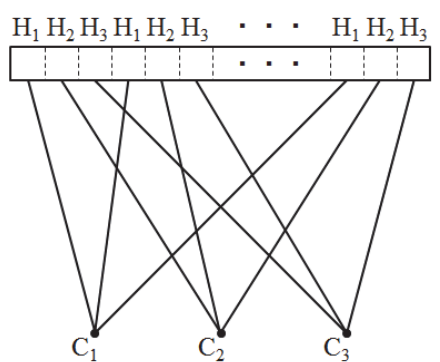

(b)

Figure 6. Structure of HOE: (a) side view, (b) top view. The whole $\mathrm{HOE}$ consists of three types of $\mathrm{HOEs}, \mathrm{H}_{1}, \mathrm{H}_{2}$ and $\mathrm{H}_{3}$ that correspond to convergent points $\mathrm{C}_{1}, \mathrm{C}_{2}$ and $\mathrm{C}_{3}$, respectively.

\section{THE IMPROVED SEE-THROUGH RETINAL PROJECTION TYPE SUPER MULTI-VIEW HMD BY USING THE TIME DIVISION PROJECTION OPTICAL SYSTEM.}

In our previous HMD, we proposed a spatial division type image projection system using a vertically-striped HOE lens array that is different for each vertical line of the parallax image as shown in Figure 6. However, since the respective parallax images were spatially divided and were projected onto the retina, the image resolution was low and the optical system was complicated. In order to overcome this problem, we propose the improved super multi-view HMD using the time division projection optical system. The proposed HMD consists of the multiple exposure holographic lens which forms some convergence points, a high frame rate display device, and a high-speed optical shutter. Figure 7 shows the structure of the multiple exposure holographic lens. In this figure, for the sake of simplicity, we limit the number of convergence points to three. In the proposed HMD, multiple parallax images are displayed by time division, and these images are converged on respective points by the multiple exposure holographic lens. The optical shutter which synchronized to the display device passes only one convergence light corresponding to the right parallax image. Therefore, proposed HMD realizes the pseudo super multi-view state. In comparison of the previous space division type HMD and the proposed time division type HMD, the advantages of the proposed HMD are the simple optical system and the high resolution viewing image. A Schematic diagram of the time division optical system of the proposed HMD is shown in Figure 8. For the sake of simplicity, we limit the number of convergence points to three. In Figure $8, \mathrm{~L}_{1}, \mathrm{~L}_{2}$ and $\mathrm{L}_{3}$ are convex lenses. The high-speed optical shutter is placed at the focal plane of the multiple exposure $\mathrm{HOE}\left(\mathrm{HOE}_{1}\right)$. Since the eyeglass lens (holographic lens $\mathrm{HOE}_{2}$ ) of the $\mathrm{HMD}$ works as a convex lens, the conjugate images $\mathrm{C}_{1}{ }^{\prime}, \mathrm{C}_{2}{ }^{\prime}$ and $\mathrm{C}_{3}{ }^{\prime}$ of the convergence points $\mathrm{C}_{1}, \mathrm{C}_{2}$ and $\mathrm{C}_{3}$ are located at the pupil, respectively. Since the conjugate images $\mathrm{C}_{1}{ }^{\prime}, \mathrm{C}_{2}{ }^{\prime}$ and $\mathrm{C}_{3}{ }^{\prime}$ correspond to the parallax image \#1, \#2 and \#3 respectively, these parallax images projected on the retina. So that the projection optical system is put in the HMD frame, the eyeglass lens $\mathrm{HOE}_{2}$ of the $\mathrm{HMD}$ should be a reflection-type hologram. However, in the evaluation experiment, the camera used as substitution for an eye interferes with the projection optical system. Thus, in the evaluation experiment, we use the transmission-type HOE lens for the eyeglass lens $\mathrm{HOE}_{2}$. In Figure 8(a), only the convergence point $\mathrm{C}_{1}$ passes through the shutter. In the same way, only the convergence point $C_{2}$ in Figure 8(b), and only the convergence point $C_{3}$ in Figure 8(c), pass through the shutter respectively. In the proposed HMD, the time division optical system works like the optical system as shown in Figure 8(d) by fast switching three states of Figure 8(a), 8(b) and 8(c) in one field. So that these parallax images corresponding to each convergence point are left as after-images on the human eye. Therefore, the super multi-view state is realized with the time division optical system. 


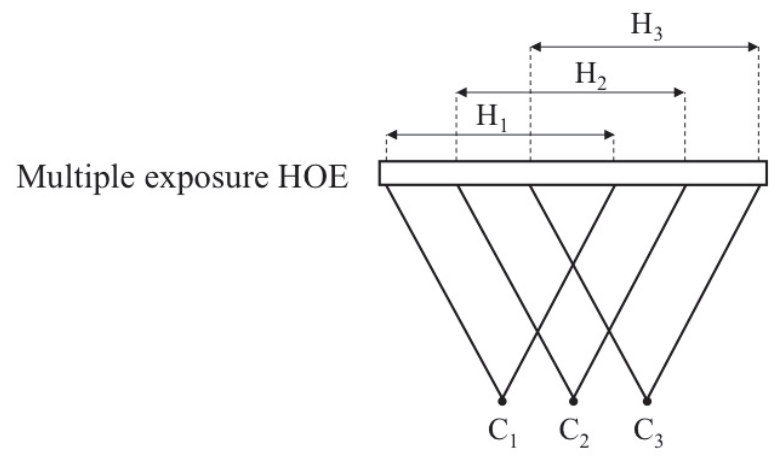

Figure 7. Structure of the multiple exposure HOE. The whole HOE consists of three types of $\mathrm{HOEs}, \mathrm{H}_{1}, \mathrm{H}_{2}$ and $\mathrm{H}_{3}$ that correspond to convergent points $\mathrm{C}_{1}, \mathrm{C}_{2}$ and $\mathrm{C}_{3}$, respectively.

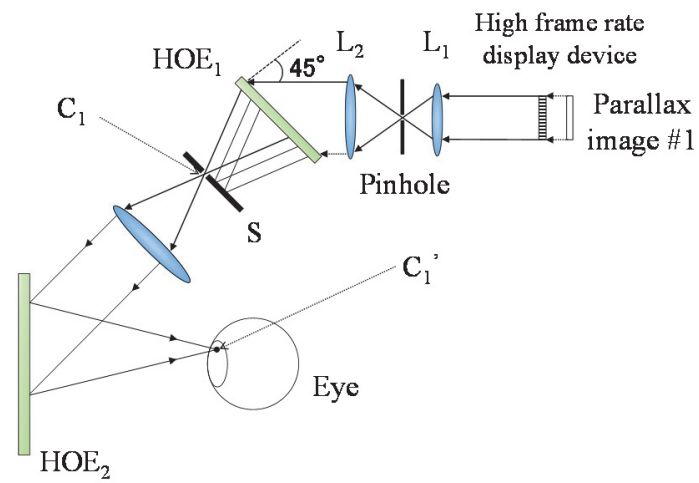

(a)

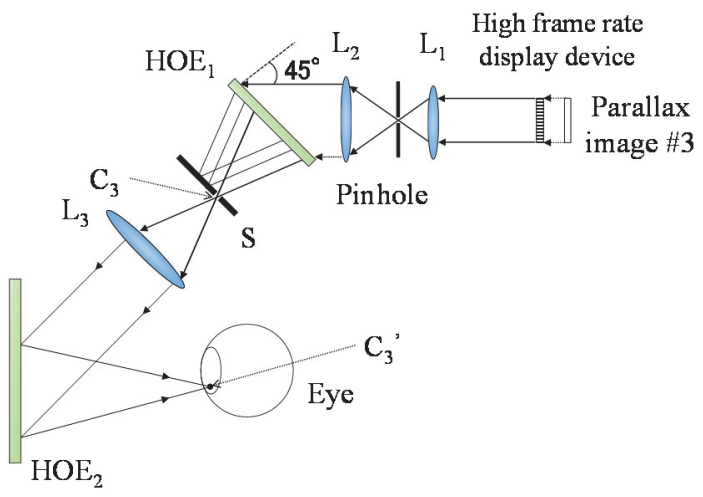

(c)

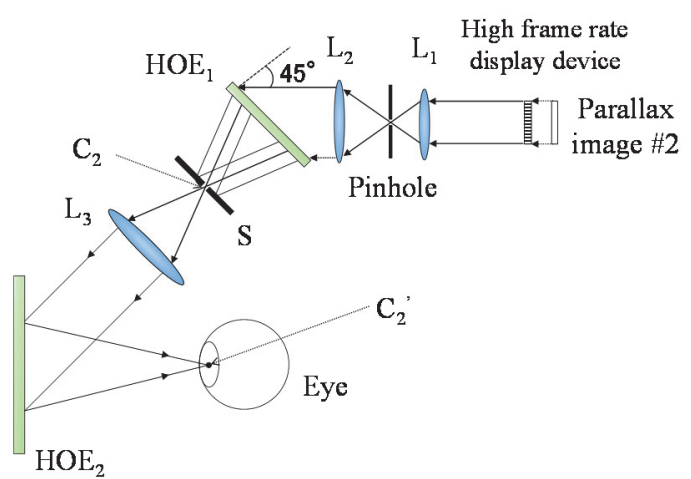

(b)

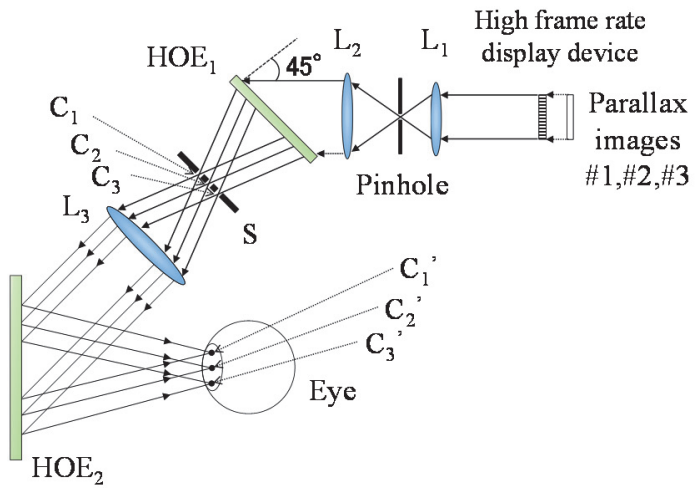

(d)

Figure 8. Schematic diagram of the proposed HMD based on the time division method with the high frame rate display device, the multiple exposure holographic lens and the high-speed optical shutter: (a) the optical system when only the convergence point $\mathrm{C}_{1}$ passes through the shutter, (b) the optical system when only the convergence point $\mathrm{C}_{2}$ passes through the shutter, (c) the optical system when only the convergence point $C_{3}$ passes through the shutter, (d) the time division optical system works like the optical system as shown in Figure (d) by fast switching three states of Figure (a), (b) and (c) in one field. 


\section{EXPERIMENTAL RESULTS}

In order to verify the effectiveness of the proposed HMD, we constructed the prototype system as shown in Figure 9. In the prototype system, the number of parallax images is three.

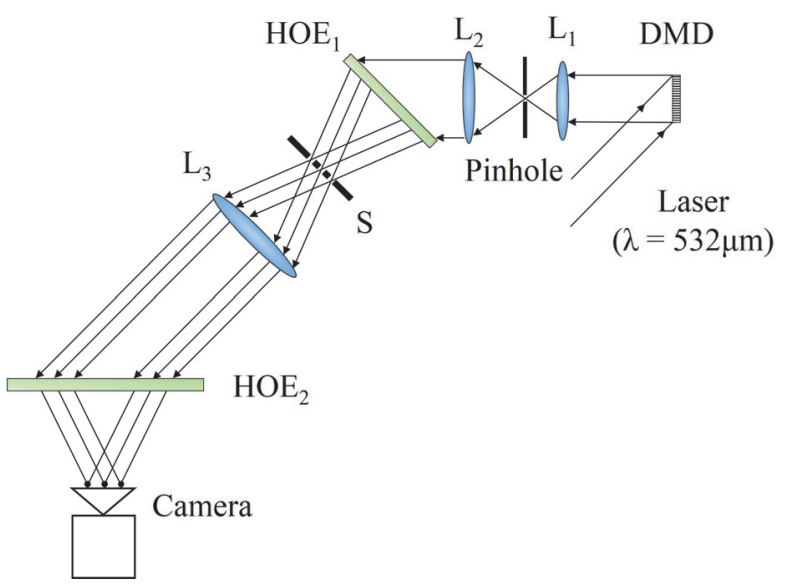

Figure 9. Prototype system.

The prototype system consists of a $\mathrm{DMD}$, a multiple exposure $\mathrm{HOE}_{1}$, a high-speed shutter, and an eyeglass lens $\mathrm{HOE}_{2}$. We used a DMD as a high frame rate display device which provides time sequential parallax images. Figure 10(a) shows the specification of the prototype multiple exposure HOE. Also, Figure 10(b) shows the specification of the eyeglass lens $\mathrm{HOE}_{2}$ which works as a convex lens.

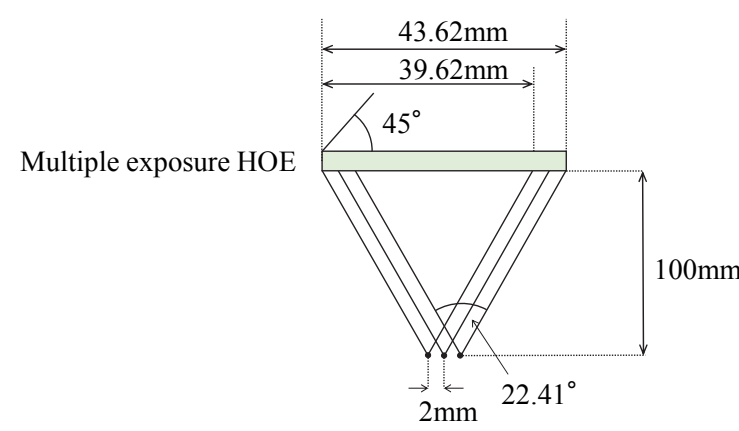

(a)

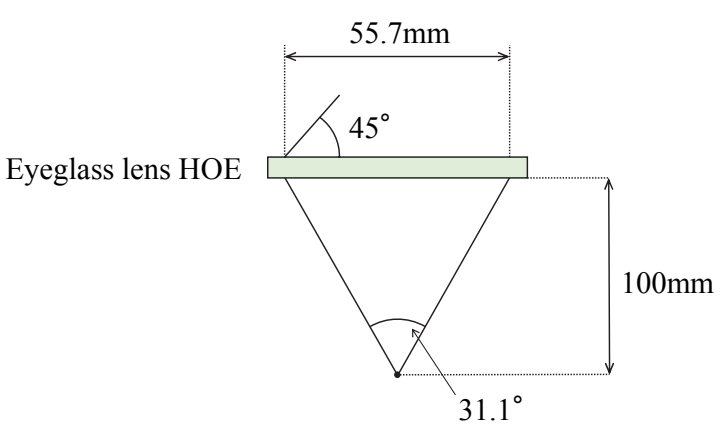

(b)

Figure 10. Specification of the prototype HOE: (a) multiple exposure HOE, (b) eyeglass lens HOE

In Figure 10, the multiple exposure HOE converges the time sequential parallax images on the DMD to the convergent points as shown in Figure 7. Since all convergent point are located on the pupil, the distance between two convergent points is $2 \mathrm{~mm}$. The focal length and the size of the multiple exposure HOE are $100 \mathrm{~mm}$ and $29.71 \mathrm{~mm} \times 39.62 \mathrm{~mm}$, respectively. And also, the focal length and the size of eyeglass lens HOE are $100 \mathrm{~mm}$ and $29.71 \mathrm{~mm} \times 55.7 \mathrm{~mm}$, respectively. We used the Digital Mirror Device (DMD) as the display device. The size of the DMD is $1024 \times 768$ and the MicroMirror Pitch is $13.68 \mu \mathrm{m}$. In the prototype system, the DMD sequentially displays three parallax images. The schematic diagram and the specifications of the high-speed optical shutter are shown in Figure 11 and Table 1. The highspeed optical shutter was realized by the wheel slit as shown in Figure 11(a) which is synchronized with the time sequential parallax images. The high-speed optical shutter has three wheel slits $\mathrm{S}_{1}, \mathrm{~S}_{2}$, and $\mathrm{S}_{3}$. Figure 11(b) shows the positional relationship between the wheel slits and the convergence points. Here, $S_{1}, S_{2}$ and $S_{3}$ correspond to the convergence points $C_{1}, C_{2}$ and $C_{3}$, respectively. As shown in Figure 11(b), when the high-speed optical shutter rotates by synchronizing with the time sequential parallax images, the convergence points $\mathrm{C}_{1}, \mathrm{C}_{2}$ and $\mathrm{C}_{3}$ sequentially pass through $\mathrm{S}_{1}, \mathrm{~S}_{2}$ and $\mathrm{S}_{3}$. 


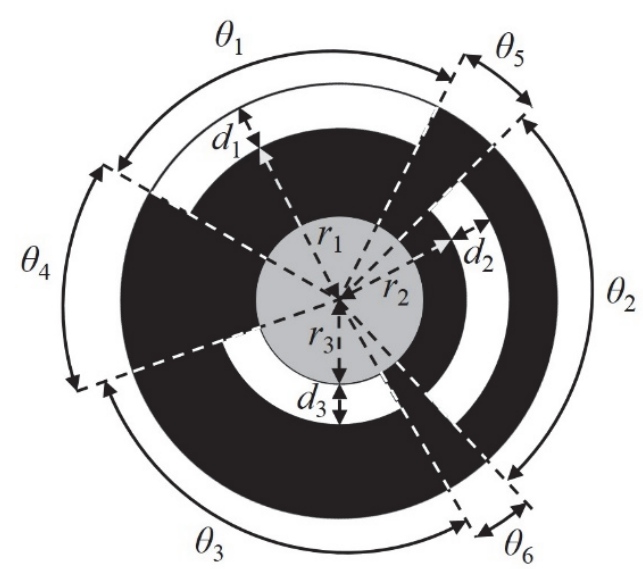

(a)

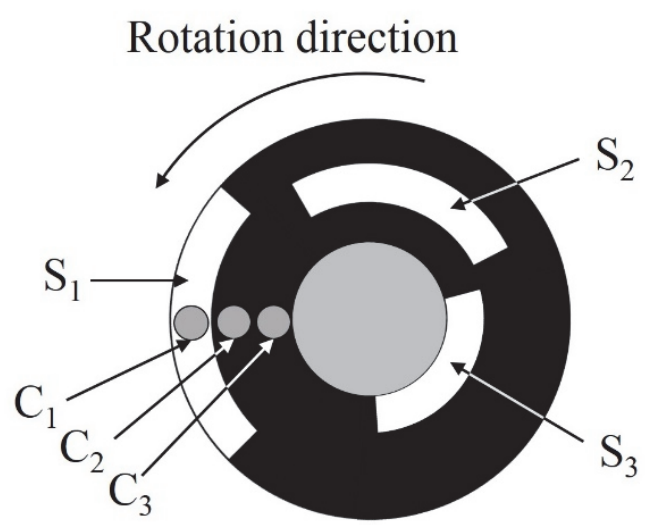

(b)

Figure 11. The high-speed optical shutter: (a) specifications of the high-speed optical shutter, (b) schematic diagram when only the convergence point $\mathrm{C}_{1}$ passes through the high-speed optical shutter.

Table 1. The specifications of the high-speed optical shutter.

\begin{tabular}{|lccccc|}
\hline \multicolumn{5}{|c}{ High-speed optical shutter Specifications } \\
\multicolumn{2}{|c}{ radius } & \multicolumn{2}{c|}{ angle } & \multicolumn{2}{l|}{ width } \\
\hline$r_{1}$ & $23 \mathrm{~mm}$ & $\theta_{1}$ & $80^{\circ}$ & $d_{1}$ & $2 \mathrm{~mm}$ \\
$r_{2}$ & $21 \mathrm{~mm}$ & $\theta_{2}$ & $96^{\circ}$ & $d_{2}$ & $2 \mathrm{~mm}$ \\
$r_{3}$ & $19 \mathrm{~mm}$ & $\theta_{3}$ & $102.6^{\circ}$ & $d_{3}$ & $2 \mathrm{~mm}$ \\
& & $\theta_{4}$ & $67.8^{\circ}$ & & \\
& & $\theta_{5}$ & $6.8^{\circ}$ & & \\
& & $\theta_{6}$ & $6.8^{\circ}$ & & \\
\hline
\end{tabular}

To verify the effectiveness of the proposed system, we used the camera as substitution for an eye and captured observed images. Since the camera was used instead of the eye, we set the eye relief of the eyeglass lens HOE to $100 \mathrm{~mm}$. Figure 12 shows the schematic diagram of the experimental setup. We displayed the virtual images which is the verticallystriped pattern that striped width is $0.6 \mathrm{~mm}$ at the distance $200 \mathrm{~mm}$ in front of the camera, and put four plate to the distance of $D_{1}=200 \mathrm{~mm}, D_{2}=500 \mathrm{~mm}, D_{3}=2000 \mathrm{~mm}$, and $D_{4}=4000 \mathrm{~mm}$ to use them in the focusing of the camera. The distance that each plate was put was indicated on four plates. And then, we confirmed the accommodation. Figure 13(a) shows the captured image which focused on the plate (the real object) at the distance of $D_{1}$ in front of the camera. In the same way, Figure 13(b), 13(c) and 13(d) show the captured images which focused on the plate at the distance of $D_{2}, D_{3}$ and $D_{4}$, respectively. As shown in Figure 13(a), the vertically-striped image at the distance of $D_{1}$ in front of the camera could be observed clear. However, from Figures 14(b), 14(c), and 14(d), we found that the vertically-striped images at the distance of $D_{2}, D_{3}$ and $D_{4}$ in front of the camera were blurred. Figure 14 shows the light intensity distribution of the observed images of Figure 13. In Figure 14(a), the contrast of light intensity distribution is $66.4 \%$, and the half value width of the light intensity distribution of each vertical stripe is $0.59 \mathrm{~mm}$, which means that all parallax images are accurately superposed at the same position and we captured the virtual image in focus. However, in Figure 14(b), the contrast is $34.0 \%$, and each parallax image are slightly shifted each other and superposed. Therefore, we captured the virtual image out of focus. In the same way, in Figures 14(c) and 14(d), since the contrast are $24.2 \%$ and $43.75 \%$ respectively, we found that the virtual images out of focus were captured. 


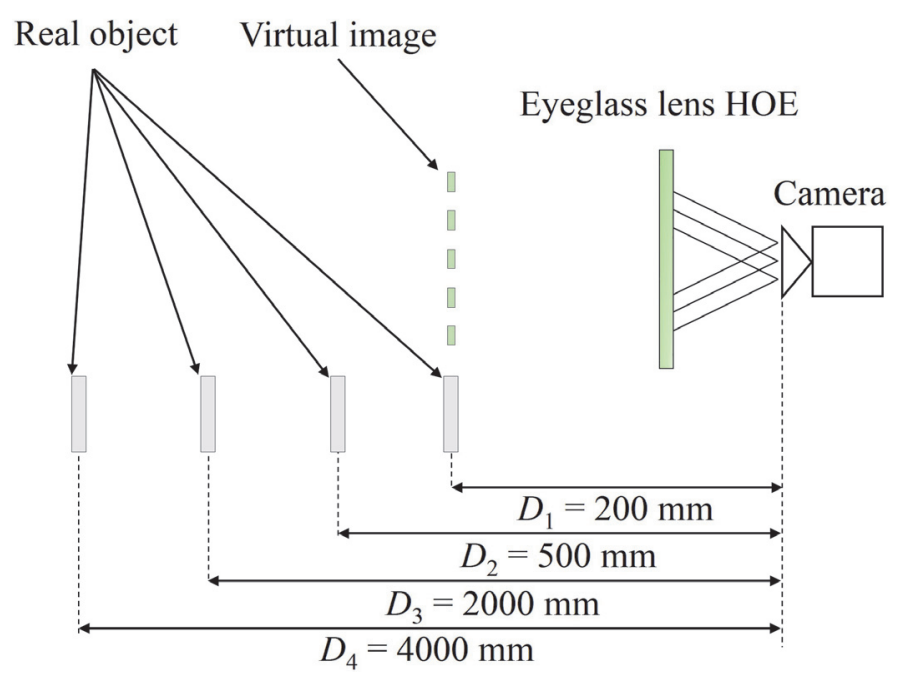

Figure 12. Schematic diagram of the experimental set up.

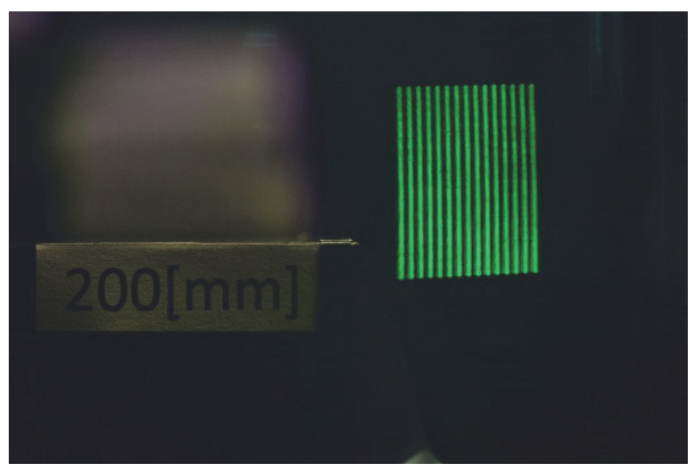

(a)

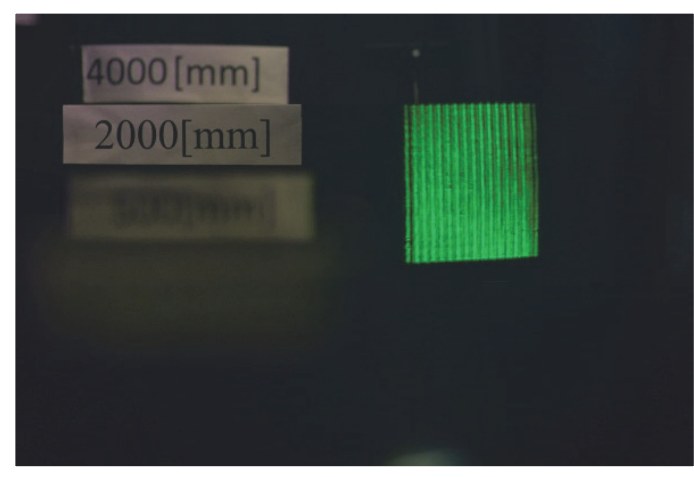

(c)

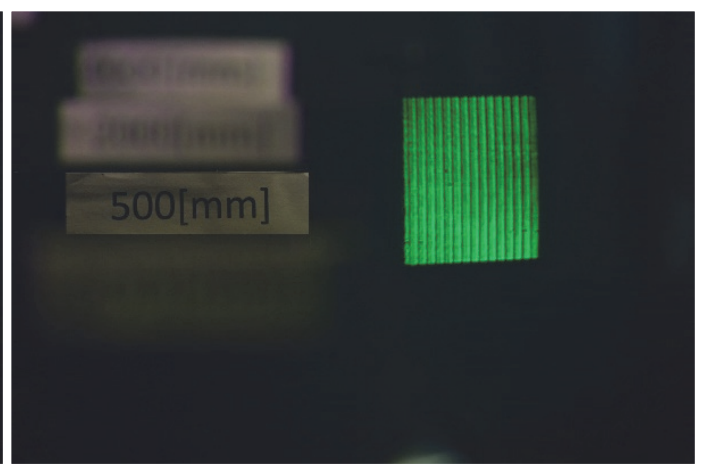

(b)

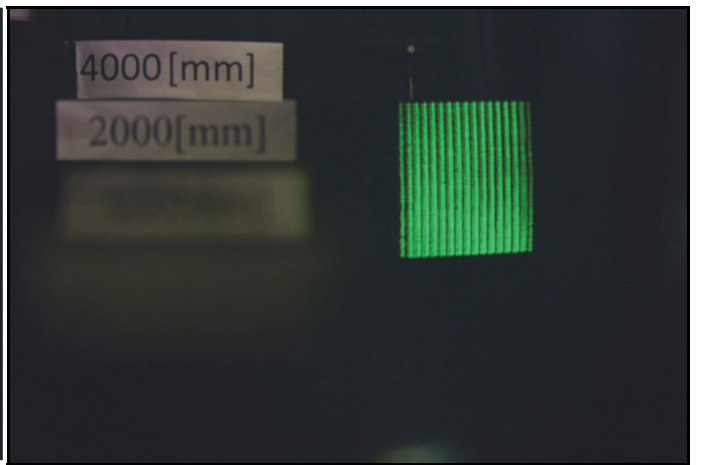

(d)

Figure 13. Observed images: (a) focused on the real object at the distance of $200 \mathrm{~mm}$, (b) focused on the real object at the distance of $500 \mathrm{~mm}$, (c) focused on the real object at the distance of $2000 \mathrm{~mm}$, (d) focused on the real object at the distance of $4000 \mathrm{~mm}$. 


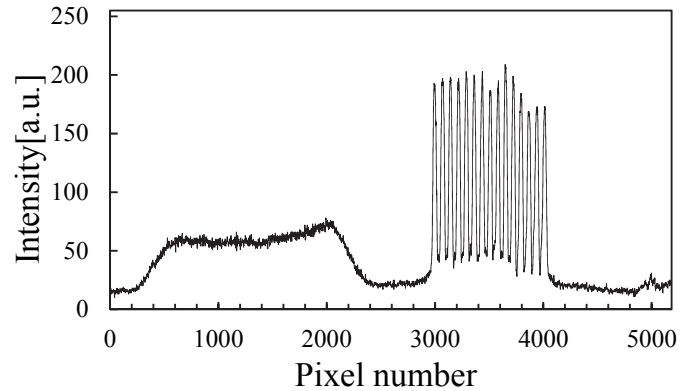

(a)

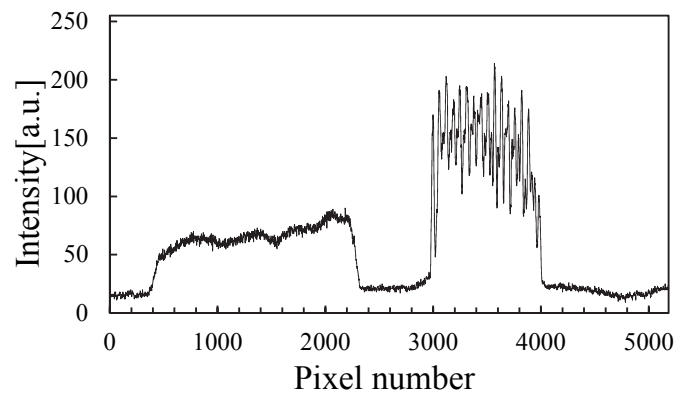

(c)

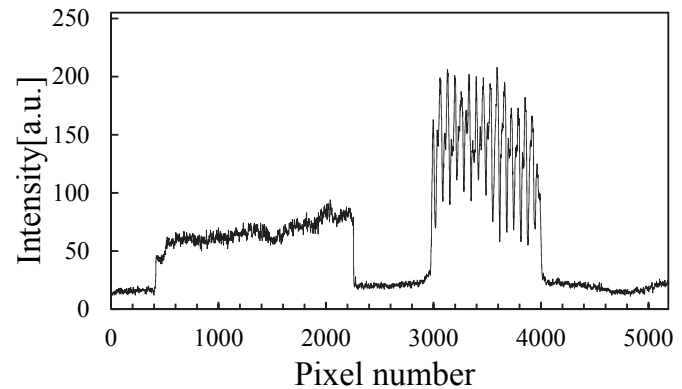

(b)

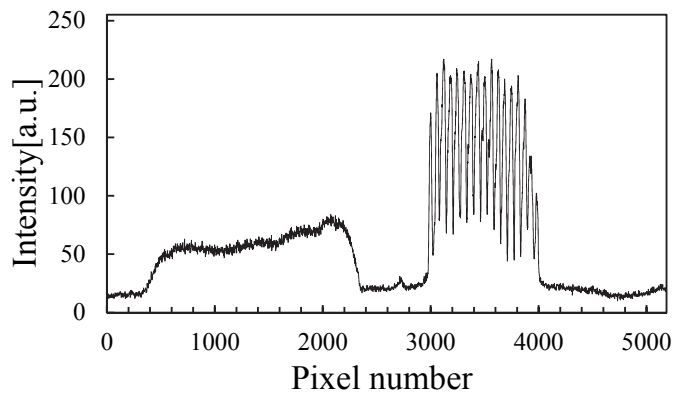

(d)

Figure 14. The light intensity distribution of the observed images: (a) focused on the real object at the distance of $200 \mathrm{~mm}$, (b) focused on the real object at the distance of $500 \mathrm{~mm}$, (c) focused on the real object at the distance of $2000 \mathrm{~mm}$, (d) focused on the real object at the distance of $4000 \mathrm{~mm}$.

Next, in the experiment setup of Figure 12, we displayed the virtual images which is the vertically-striped pattern that striped width is $6 \mathrm{~mm}$ at the distance $2000 \mathrm{~mm}$ in front of the camera, and captured observed image. Figure 15 shows the observed image which focused on the plate at the distance of $2000 \mathrm{~mm}$ in front of the camera and the light intensity distribution of the observed image.

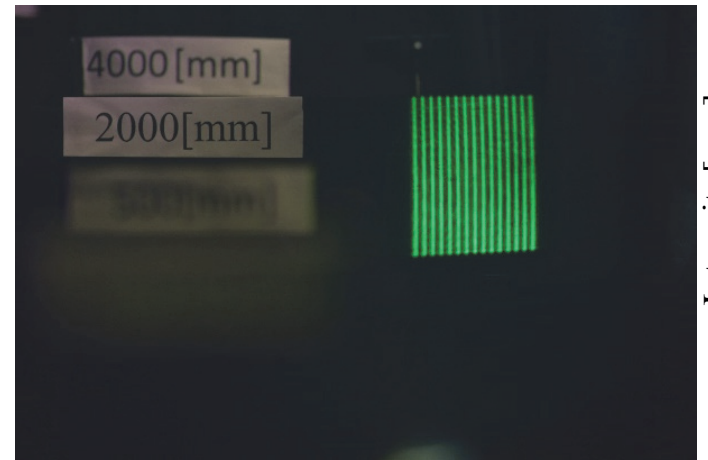

(a)

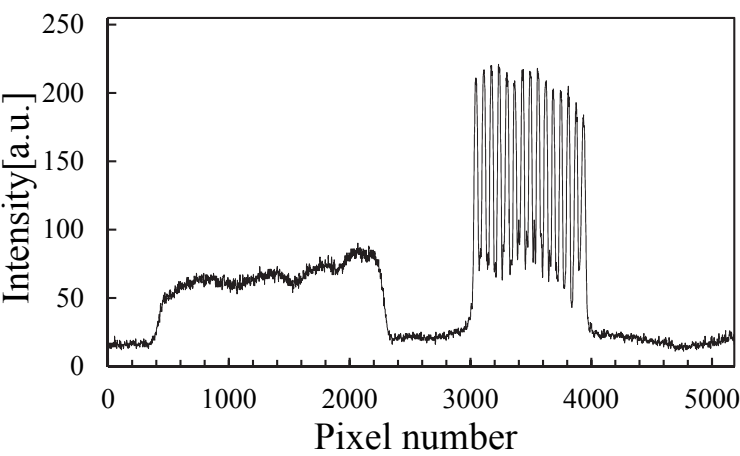

(b)

Figure 15. Observed image which focused on the plate at the distance of $2000 \mathrm{~mm}$ : (a) the observed image, (b) the light intensity distribution of the observed image.

As shown in Figure 15 (a), the vertically-striped image at the distance of $D_{3}$ in front of the camera could be observed clear. In Figure 15(b), the contrast of light intensity distribution is $55.6 \%$, and the half value width of the light intensity distribution of each vertical stripe is $0.59 \mathrm{~mm}$, which means that all parallax images are accurately superposed at the same position and we captured the virtual image in focus. From the above, we found that the natural stimulus for the accommodation was reproduced by the proposed HMD. 


\section{CONCLUSION}

In conclusion, a retinal projection type super multi-view 3D head-mounted display using the time division projection optical system was proposed. The proposed HMD has a higher resolution compared with the conventional super multiview HMD using space division projection optical system. Also the HOE which is used the proposed HMD has a simple structure and is made by a simple method as compared with the HOE which is used the conventional super multi-view optical HMD using space division projection optical system. In the prototype system, since the number of projected parallax images is three, the smooth motion parallax provided horizontally. The proposed HMD displays the virtual image at the distance within the ability for focusing on the eye. To verify the effectiveness of the proposed HMD, we displayed virtual images at the distance from $20 \mathrm{~cm}$ to $200 \mathrm{~cm}$ in front of the camera, and confirmed the accommodation. The proposed HMD enables a precise superposition of virtual 3D images on the real scene.

\section{ACKNOWLEDGEMENT}

This work was partially supported by JSPS KAKENHI Grant Number 15 K00281.

\section{REFERENCES}

[1] Tamura, H. and Ohta, V., [Mixed Reality], Ohmsha (1999).

[2] Burdea, G. and Coiffet, P., [Virtual Reality Technology], John Wiley \& Sons (1994).

[3] Takahashi, H., Ito, Y., Nakata, S. and Yamada, Y., "Retinal projection type super multi-view head-mounted display," Proc. SPIE-IS\&T Electronic Imaging, 9012, 90120L1-9012L6 (2014).

[4] Takatsuka, Y., Yoshimoto, K. and Takahashi, H., "Retinal projection type 3D head-mounted display using an HOE lens array," Proc. SPIE-IS\&T Electronic Imaging 2016, SDA-428.1-SDA-428.6 (2016).

[5] Emoto, T., Konda, T., Yoshimoto, K. and Takahashi, H., "Analysis of retinal images for retinal projection type super multi-view 3D head-mounted display," Proc. IS\&T Electronic Imaging 2017, 188-193 (2017).

[6] Kajiki, Y., Yoshikawa, H. and Honda, T., "Ocular Accommodation by Super multi-view Stereogram and 45View Stereoscopic Display," Proc. IDW'96, 2, 489-492 (1996).

[7] Westheimer, G., "The Maxwellian View," Vision Res. 6, 669-682 (1966).

[8] Takahashi, H. and Hirooka, S., "Stereoscopic See-Through Retinal Projection Head-Mounted Display," Proc. SPIE 6803, 68031N1-68031N8 (2008).

[9] Takaichi, K. and Takahashi, H., "Multi-Color See-Through Retinal Projection Head-Mounted Display," ICIC Express Letters 6(5), 1291-1296 (2012).

[10] Ito, Y., Takaichi, K. and Takahashi, H., "See-Through Retinal Projection Head-Mounted Display by using the Extended Maxwellian View," ICIC Express Letters 7(6), 1755-1760 (2013). 